\title{
Liderazgo de la dirección y participación de los trabajadores en el ámbito de la seguridad y salud en el trabajo
}

\author{
Leadership management and participation of workers in the scope of the health \\ and safety at work
}

Jaume Figueras Esgleas

Director de Prevención de Croda Ibérica SA. España.

\section{Correspondencia}

Jaume Figueras Esgleas

Director de Prevención de Croda Ibérica SA. España.

Correo electrónico: jaume.figueras@croda.com

\section{ResUmen}

Creación e implantación de un grupo de participación plena en la toma de decisiones en seguridad y salud laboral, liderado por la Dirección, y formado por: trabajadores, delegados de prevención, técnicos responsables de la seguridad y salud de la empresa (Servicio de Prevención Propio) y técnicos representantes de la autoridad laboral de la administración de la Generalidad de Cataluña -Departamento de Empresa y Empleo- con la voluntad de potenciar la participación de los trabajadores y de sus representantes y de incrementar una mejor integración de la prevención en todos los niveles jerárquicos de la empresa.

A partir de las observaciones preventivas realizadas por los trabajadores que ocupan los lugares de trabajo, las visitas de campo y las reuniones realizadas por el grupo de participación plena se analizaron diferentes tareas de las áreas de trabajo adoptando soluciones de mejora de las condiciones de seguridad y salud en el trabajo.

Todas estas mejoras implantadas han propiciado una mayor integración de los trabajadores de la empresa en la organización, cuya consecuencia ha generado una mejora del clima laboral y una cultura positiva de seguridad y salud, una mayor implicación, participación y compromiso de toda la organización y un liderazgo activo en seguridad y salud de la dirección de la empresa consiguiendo ser una empresa más competitiva, se fomente la innovación en materia preventiva y, como resultado, ser una empresa socialmente responsable y sostenible.

Palabras clave: Liderazgo, Participación, Compromiso, Implicación, Trabajo en equipo.

Abstract

Creating and implementing a dedicated participation group to take decisions regarding Health and safety. This group, led by management, consists of workers, safety representatives, company Health and Safety technicians and representatives of the department of Labour (Generalidad de Cataluña). The aim is to enhance the participation of workers and their representatives and to increase a better integration of prevention at all levels of hierarchy in the company.

From preventive observations made by workers in their own workplaces, site visits and meetings held by the participation group, different tasks were analyzed in the workplaces to adopt improvement solutions for the Health and Safety conditions at work. 
All these improvements implemented have led to greater integration of company employees in the organization. The consequence has generated an improved working environment and a positive culture of health and safety, greater involvement, participation and commitment of the entire organization and active leadership in health and safety of the company management, making for a more competitive company which promotes innovation in preventive matters and as result, to be a socially responsible and sustainable company.

Keywords: Leadership, Participation, Commitment, Involvement, Teamwork. 


\section{INTRODUCCIÓN}

Croda Ibérica SA - Mevisa Site, es una Sociedad Unipersonal, inscrita en el Registro Mercantil de Barcelona, Tomo 31274, folio 29, hoja número B-192684, CIF. A 61803136, dedicada a la fabricación y comercialización de Productos Químicos en general, que forma parte de Croda Ibérica S.A. que es la filial española de Croda International Plc, multinacional inglesa con sede en Cowick Hall, Snaith, East Yorkshire.

Con domicilio en Ctra. C-35, Km 72 (Hostalric-Blanes), perteneciendo al municipio de Fogars de la Selva, provincia de Barcelona.

Croda Ibérica S.A. - Mevisa Site diseña y produce productos tensioactivos que se utilizan en múltiples aplicaciones como la industria textil, papel, curtidos, farmacia, cosmética y agricultura, con un volumen anual de fabricación de 37.000 Toneladas.

\section{PRL en la empresa}

Croda Ibérica S.A.-Mevisa Site lleva trabajando con Técnicas de Prevención desde sus inicios en 1.975, durante los cuales ha ido elaborando y poniendo en funcionamiento normativas de Prevención que le han permitido mantener unos estándares aceptables.

Pero es en el año 1.993 cuando se implanta un Sistema de Gestión de la Prevención basado en los principios del Control Total de Pérdidas y de los Sistemas de Calidad que infunden a la organización, una visión más amplia de como puede ser utilizado el sistema como herramienta de gestión para el Control de costes de nuestra empresa influyendo también, en otras áreas de suma importancia (Calidad, Productividad...).

En el año 2.006 certifica el Sistema de Gestión basado en la certificación OHSAS 18001 con la finalidad de mejorar y dando así respuesta tanto a la norma como a la legislación en prevención.

La empresa con el afán de optimizar la eficiencia y eficacia del sistema implantado constituye un Sistema de Gestión Integrado en toda la Organización basado en la mejora continua.

Con el fin de conseguir este propósito, la empresa quiere lograr entornos de trabajo seguros y saludables, por este motivo integra la prevención en la gestión de la actividad diaria, de la misma manera y con los mismos esfuerzos dedicados a la calidad, productividad, clientes y costes para llegar a ser una empresa competitiva, líder y referente en materia de seguridad.

El Sistema de Gestión de Seguridad de Croda Ibérica S.A. está integrado dentro de todos los Sistemas de Gestión de la Empresa (Calidad; Seguridad y Salud Laboral; Medio Ambiente; Ingeniería; Responsabilidad Social Corporativa) que formalmente está documentado a partir de un Manual de Sistema de Gestión.

A partir de este Manual, cada uno de los Sistemas de Gestión que lo integran establece su propio Manual de Gestión, en el que se describe la Política específica del Sistema que representa, así como la autoridad y recursos asignados, su control documental, la Organización necesaria para su actividad, los estándares y normas que son aplicadas y/o tomadas como referencia de cumplimiento y los elementos del Sistema de Gestión que lo configuran; todo ello dentro de un círculo de mejora continua.

La prevención así planteada se convierte en una actividad que se fundamenta en el compromiso y liderazgo de la dirección y de todos los mandos intermedios, requiriendo la participación e implicación de todas las personas ya sean propias o ajenas, con el fin de desarrollar una cultura preventiva sólida donde todos actuemos siempre evitando que cualquier persona pueda accidentarse. 


\section{Proyecto particular}

\section{Desarrollo}

En el marco de este proyecto se ha creado en la empresa Croda Ibérica S.A. un grupo de participación plena, liderado por la Dirección, en la toma de decisiones en seguridad y salud laboral que ha analizado, con implicación, participación y compromiso de todos los actores implicados, las diferentes tareas y lugares de trabajo de la empresa.

Se ha estructurado de la siguiente manera:

a) En primer lugar se presentó el proyecto a todos los niveles de la empresa (Dirección, Comité de Empresa, Delegados de prevención y Comité de Dirección).

b) Se creó un grupo de participación plena en la toma de decisiones en seguridad y salud laboral formado por: trabajadores, delegados de prevención, dirección de la empresa, técnicos responsables de la seguridad y salud de la empresa (servicio de prevención propio) y técnicos representantes de la autoridad laboral de la administración de la Generalidad de Cataluña -Departamento de Empresa y Empleo, para canalizar, desarrollar y liderar el proyecto.

c) Este equipo de trabajo mantuvo una reunión inicial para definir la estrategia de trabajo y la metodología a seguir tomando como base de trabajo los siguientes documentos: la evaluación de riesgos laborales, los incidentes, las observaciones preventivas elaboradas por los propios trabajadores en las diferentes áreas de trabajo, las auditorías internas y externas, las reuniones del Comité de Seguridad y Salud Laboral, los procedimientos y las instrucciones de trabajo.

Partiendo del análisis de los documentos mencionados anteriormente, se confeccionó un listado de actuaciones a valorar.

d) A partir de este punto se inició el proyecto propiamente dicho, cual se ha desarrollado de la siguiente manera:

- Segregación de las actuaciones por áreas de trabajo.

- Visitas programadas a las áreas de trabajo, con el grupo de participación plena, para valorar y analizar la viabilidad de las actuaciones propuestas.

- Una vez valoradas las actuaciones a llevar a cabo, se establecieron responsables y plazos para su ejecución, siempre siguiendo los procedimientos internos del Sistema de Gestión de la Seguridad implantados en la empresa.

- El alcance de este proyecto ha englobado todas las áreas de la empresa, Producción, Mantenimiento, Almacén, Laboratorio y Oficinas, teniendo en cuenta tanto a los trabajadores propios como a los contratistas.

- La dirección de la empresa ha participado activamente en las reuniones relevantes del grupo de trabajo de participación plena.

\section{Problemas detectados}

Se analizaron diferentes tareas de las áreas de trabajo, destacando las siguientes:

1. Manipulación manual de cajas de producto acabado en la zona de pastilladora de la Nave E y la escamadora de la Nave C, y manipulación manual de sacos de materia prima para la carga de los reactores en la línea 4 de la Nave E.

2. Accesos de los operarios y empresas contratistas a tejados, tanques de almacenamiento y cisternas.

3. Intervención de los operarios en trabajos de carga y descarga de productos líquidos en las plantas de producción y manipulación de éstos en las áreas de laboratorio de planta.

4. Intervención de los operarios en la manipulación manual de bidones así como en las tareas de limpieza en la zona de descarga de carbones procedentes de filtraje.

5. Intervención del operario en la manipulación de válvulas de purga. 


\section{Implantación}

Se adoptaron las soluciones que a continuación detallamos:

1. Se han instalado equipos de manipulación neumática ingrávidos en las áreas donde se había identificado el riesgo.

2. Se han instalado líneas de vida en las siguientes áreas:

- Todas las zonas de carga y descarga de cisternas.

- Todos los accesos a los tanques de almacenamiento.

- Todos los tejados de la factoría.

Se ha dado la formación necesaria a todos los trabajadores para la correcta utilización de estos equipos y la realización de trabajos en altura.

3. Se ha llevado a cabo un proyecto específico para esta área que ha consistido en las siguientes mejoras:

- Construcción de drenajes a lo largo del área de descarga.

- Se le ha dado el desnivel oportuno al suelo para favorecer la contención del producto en caso de derrame.

- Se ha colocado un suelo de baldosas a lo largo de toda la zona de descarga para favorecer su limpieza.

- Se ha aplicado la metodología LOTO (Lockout-Tagout) para la descarga de los camiones, para evitar posibles accidentes. Con este procedimiento se consigna la llave del camión hasta que el responsable de la descarga dé la orden que se puede retirar el vehículo en condiciones de seguridad.

4. Para cada uno de los filtros se ha instalado un camino de rodillos para mover los palets (en cada palet se disponen 4 bidones), así como la instalación de tolvas móviles (para ir llenando los 4 bidones sin necesidad de manipularlos). Los palets son manipulados directamente con las carretillas elevadoras. Se han colocado baldosas en toda el área y se ha instalado un equipo de limpieza con agua caliente a presión.

5. Se han sustituido las válvulas de purga manuales por válvulas manuales de retorno automático en todos los equipos identificados con este riesgo.

\section{Eficacia y resultados}

Los resultados conseguidos con la implantación de este proyecto son los que se definen a continuación:

- Mejora material y técnica efectiva de las condiciones de seguridad y salud, eliminando y, en algunos casos, minimizando el riesgo identificado.

- Mejora en la reducción de los incidentes reportados.

- Mejora en los costes de producción de la empresa.

- Mejora los canales de información, consulta, diálogo y resolución de conflictos entre los diferentes actores que la constituyen.

- Mayor integración de los trabajadores de la empresa en la organización, cuya consecuencia ha generado una mejora del clima laboral y una cultura positiva de seguridad y salud.

- Mejora de la implicación, participación y compromiso de la dirección de la empresa, del servicio de prevención propio y de toda la organización.

- Liderazgo activo en seguridad y salud de la dirección de la empresa y del servicio de prevención propio.

- Participación directa y activa de los trabajadores y sus representantes en el proceso de evaluación, análisis, decisión y planificación/implantación de las medidas correctoras. 
- Mejora de la concienciación y cooperación activa de los trabajadores como consecuencia de un cambio de actitud promovido por una mejor comunicación entre todos los estamentos de la organización.

- Mejora de la cultura preventiva como vehículo para conseguir espacios de trabajo más saludables.

- Mejora de la relación-comunicación bilateral entre la empresa y la autoridad laboral de la administración, actuando ésta como asesor externo en la ejecución del proyecto.

\section{Factores de éxito}

Han contribuido al éxito del proyecto:

- La buena disposición, colaboración e implicación activa de todos los trabajadores y sus representantes (delegados de prevención, comité de seguridad y salud, comité de empresa).

- El compromiso de la dirección y la política de prevención existente en la empresa Croda Ibérica SA, para canalizar, desarrollar y liderar el proyecto.

- El establecimiento de objetivos alcanzables y medibles.

- El trabajo constante y diario del servicio de prevención propio de la empresa, el asesoramiento externo de los técnicos representantes de la autoridad laboral de la administración de la Generalidad de Cataluña -Departamento de Empresa y Empleo para la dinamización del proyecto.

\section{AGRADECIMIENTOS}

Delegados de Prevención, técnicos representantes de la autoridad laboral de la administración de la Generalidad de Cataluña -Departamento de Empresa y Empleo y a todos los miembros de la Organización de Croda Ibérica, SA. 